\title{
The fraction of sensitization among lung transplant recipients in a transplant center in Japan
}

Sakiko Kumata ${ }^{1 \dagger}$, Takashi Hirama ${ }^{1,2^{*}+} \mathbb{D}$, Yui Watanabe ${ }^{1}$, Hisashi Oishi', Hiromichi Niikawa', Miki Akiba ${ }^{2}$, Jussi Tikkanen ${ }^{3}$ and Yoshinori Okada ${ }^{1,2}$ (B)

\begin{abstract}
Background: Anti-human leukocyte antigen (HLA) antibody testing was approved by the Japanese government in 2018. As such, there was no longitudinal data regarding the HLA-sensitization of lung transplant (LTX) patients in Japan. We therefore set out to measure anti-HLA antibodies from all our LTX patients during their annual follow-up to characterize the sensitization status in the Japanese population.

Methods: The cross-sectional study was conducted for consecutive LTX recipients who underwent transplantation from January 2000 to January 2020 at Tohoku University Hospital (TUH). The serum from the recipients was screened for anti-HLA antibody with the panel-reactive assay (PRA) and the donor-specific antibodies (DSA).

Results: Sensitization was reviewed in 93 LTX recipients, showing 23 positive (24.7\%) and 70 negative (75.3\%) PRA. More sensitized recipients were found in recent transplantations $(60.9 \%(14 / 23), \leq 5$ years post LTX) than in older transplantations (17.4\% (4/23), 5-10 years or 21.7\% (5/23), $\geq 10$ years post LTX) $(p=0.04)$. Even fewer recipients had DSA (5.4\%, 5/93), among whom 4/5 (80\%) were recently transplanted.

Conclusion: The rate of PRA positive LTX recipients in our population was lower compared with those in previous reports from US and Europe. More sensitized LTRs were found in recent transplantations than the older cohort, and DSA was identified primarily in the recent recipients. Due to several limitations, it is still unclear whether the sensitization would be related the development of CLAD or survival, yet this study would be fundamental to the future anti-HLA body study in Japanese population.
\end{abstract}

Keywords: Lung transplant, Panel-reactive assay (PRA), Donor-specific antibodies (DSA), Japan, Human leukocyte antigen (HLA), Chronic lung allograft dysfunction (CLAD)

\footnotetext{
* Correspondence: thirama@icloud.com

${ }^{\dagger}$ Sakiko Kumata and Takashi Hirama contributed equally to this work.

'Department of Thoracic Surgery, Institute of Development, Aging and

Cancer, Tohoku University, Sendai, Miyagi, Japan

${ }^{2}$ Division of Organ Transplantation, Tohoku University Hospital, 4-1

Seiryomachi, Sendai, Miyagi 980-8574, Japan

Full list of author information is available at the end of the article
}

C C The Author(s). 2020 Open Access This article is licensed under a Creative Commons Attribution 4.0 International License, which permits use, sharing, adaptation, distribution and reproduction in any medium or format, as long as you give appropriate credit to the original author(s) and the source, provide a link to the Creative Commons licence, and indicate if changes were made. The images or other third party material in this article are included in the article's Creative Commons licence, unless indicated otherwise in a credit line to the material. If material is not included in the article's Creative Commons licence and your intended use is not permitted by statutory regulation or exceeds the permitted use, you will need to obtain permission directly from the copyright holder. To view a copy of this licence, visit http://creativecommons.org/licenses/by/4.0/ The Creative Commons Public Domain Dedication waiver (http://creativecommons.org/publicdomain/zero/1.0/) applies to the data made available in this article, unless otherwise stated in a credit line to the data. 


\section{Backgrond}

Japan has taken steps towards increasing number of donors and recipients and lung transplantation (LTX) has become a standard clinical practice for patients with advanced respiratory disease. Due to severe shortage of donor organs, the country has a unique profile in transplantation [1] such that the number of unilateral LTX outnumbers that of the bilateral. In addition, an upper age limit has been set for the registry of transplantation (younger than 60 years old for the unilateral transplant and 55 for the bilateral) and living-donor lobar LTX is considered an ethically justifiable procedure and still accounts for $10-20 \%$ of transplants [1].

The role of donor-specific antibodies (DSA) against class I and II human leukocyte antigen (HLA) has gained increasing attention in LTX. Development of DSA is associated with increased chronic lung allograft dysfunction (CLAD) and morbidity and mortality in LTX recipients [2-4]. In Japan, there is no data regarding the HLA-sensitization of LTX patients until 2018 as antiHLA antibody testing was not covered by the government. Therefore, there is no existing knowledge on the postoperative sensitization status of LTX recipients in Japan.

In this study, we set out to serially measure anti-HLA antibodies from all our LTX patients during their yearly follow-up to characterize the sensitization status in our post LTX population.

\section{Methods}

\section{Study design and data collection}

All LTX recipients (LTR) who underwent transplantation from January 2000 to January 2020 at Tohoku University Hospital (TUH) were consecutively included in the analysis. The cross-sectional study was conducted between January 2019 and January 2020, when the antiHLA antibody was measured in LTRs who visited the annual assessment. LTRs were fully assessed at months 3 and 6 post-transplant and thereafter annually. Participants' clinical details at the time of transplant were reviewed from the medical records, and the current status of lung function was assessed when the blood sample was collected. The study protocol was reviewed by the Institutional Review Board (IRB) at Tohoku University Hospital (IRB number 2019-01-54). In light of the retrospective design, the requirement of informed consent was waived. We disclosed information on the implementation of the research and ensured the opportunity for research subjects to refuse participation by posting the information disclosure materials approved by the Ethics Committee on the website of the Graduate School of Medicine, Tohoku University.

\section{Anti-HLA antibody test}

The serum from the recipients was screened for antiHLA antibody with Flow PRA (One Lambda, Inc., Canoga Park, CA). The LTR was considered sensitized when the panel-reactive assay (PRA) was positive (greater than 0\%) and regarded unsensitized when PRA was negative. The sensitized recipients were further tested for donor-specific antibody (DSA) with LABScreen single-antigen beads (One Lambda, Inc.). The positive DSA was defined by mean fluorescence intensity (MFI) units greater than 1000 or more. The complement-binding function in DSA was measured with $\mathrm{C} 1 \mathrm{q}$ Screen (One Lambda Inc.). The test for antiHLA antibody was done in ReproCELL Japan Inc. Yokohama.

\section{HLA testing in Japan}

Pre-transplant PRA was not covered by the government and not routinely measured in LTX candidates so far. At the time of transplant, HLA typing at A, B and DRB1 loci was measured in both a donor and recipients, with additional loci typing in our center since 2018. Prior to transplant performed, complement dependent cytotoxicity (CDC) $\mathrm{T}$ cell cross-match using current or historic sera needs to be carried out without dithiothreitol (DTT) reduction, which is applied for transplant decision-making; the candidates must be negative in CDC cross-match. For the time being, CDC B cell crossmatch and flow cytometry cross-match are not routinely performed in Japan. As HLA typing at HLA-C and DQB1 in donors has not been measured until 2018, Cwand DQ-DSA were not fully assessed in this cohort.

\section{Immunosuppression protocol and acute allograft rejection}

Induction of immunosuppression was achieved using basiliximab. Maintenance of immunosuppression consists of tacrolimus targeting the trough level of 10-14 $\mathrm{ng} / \mathrm{ml}$ for the first 6 months, $9-13 \mathrm{ng} / \mathrm{ml}$ up to 12 months and $8-10 \mathrm{ng} / \mathrm{ml}$ thereafter, mycophenolic acid with $1000 \mathrm{mg}$ for $<50 \mathrm{~kg}$ or $1500 \mathrm{mg} \geq 50 \mathrm{~kg}$ as tolerated and prednisone $1.0 \mathrm{mg} / \mathrm{kg}$ for the first 4 days, followed by $0.8 \mathrm{mg} / \mathrm{kg}$ for 4 days and gradually tapered to $5 \mathrm{mg}$. When LTRs could not tolerate tacrolimus or mycophenolic acid, cyclosporine or azathioprine is the alternative, respectively. Acute allograft rejection, considered when acutely dropped lung function without episodes of infection or mechanical factors such as airway stenosis, pleural effusion or native lung hyperinflation, was treated with bolus of methylprednisolone $500 \mathrm{mg}$ for 3 consecutive days, followed by tapering doses of prednisone back to $5 \mathrm{mg}$. Surveillance bronchoscopy was not routinely scheduled at TUH. 


\section{Definition of CLAD}

Chronic lung allograft dysfunction (CLAD) was defined as a substantial $(\geq 20 \%)$ and persistent ( $\geq 3$ months) decline in FEV1 from the baseline which is the mean of the best 2 post-operative FEV1 (taken $>3$ weeks apart) in a lung transplant recipient (LTR) survived $\geq 3$ months [5].

\section{Definition of CLAD subtypes}

Restrictive allograft syndrome (RAS) was defined by a concomitant $(\geq 10 \%)$ decline in TLC from the baseline which is the mean of the best 2 post-operative TLC (taken $>3$ weeks apart) and persistent opacities on thoracic CT among those who developed CLAD [6]. Bronchiolitis obliterans syndrome (BOS) is defined by the presence of the airflow limitation (FEV1/FVC ratio < $0.70)$ among those who developed CLAD. Mixed phenotype is defined by both features of RAS ( $\geq 10 \%$ decline of TLC) and BOS (FEV1/FVC ratio $<0.70)$ among LTRs with CLAD. Undefined is considered when phenotype of CLAD is difficult to be categorized among patients with CLAD [5].

\section{Statistical analysis}

Categoric variables were reported as percentages, and continuous variables as medians (interquartile range $(\mathrm{IQR}))$ as appropriate. Differences between groups were assessed with chi-square or Fisher's exact tests for categoric variables and Mann Whitney test (two groups) or Kruskal-Wallis (three groups) as appropriate for continuous variables. Statistical analyses were performed using EZR (Saitama Medical Center, Jichi Medical University, Omiya, Japan), which is a graphical user interface for $\mathrm{R}$ (The $\mathrm{R}$ Foundation for Statistical Computing,
Vienna, Austria), where a two-sided $P$ value was calculated. Graph generation was performed with GraphPad Prism 6.0 (GraphPad Software, Inc., La Jolla, CA).

\section{Results}

Up until January 2020, 760 LTX comprising 526 deceased-donor and 234 living-donor have been performed in Japan, of which TUH accounted for $17.1 \%$ (130/760). Of those, LTRs who have passed away $(n=$ 30) and could not participated in the annual assessment $(n=5)$ were excluded from the study. The initial transplantation in re-transplanted recipients were also excluded $(n=2)$ (Fig. 1). Sensitization was then reviewed in the rest of 93 patients, showing 23 positive (24.7\%) and 70 negative (75.3\%) PRA. The principal analyses were to compare PRA+ $(n=23)$ with PRA- recipients $(n=70)$, summarized in the main tables. The secondary analyses were, although the number was small, to see recipients who developed the donor-specific antibody $(\mathrm{DSA})(n=5)$, carried non-DSA anti-HLA antibody $(n=$ 18) and did not possess anti-HLA antibody $(n=70)$, summarized in supplemental files.

The patients' characteristics at the time of transplantation were summarized (Table 1). The median age in LTRs at the time of transplantation was 42 (IQR 32-50), and $57.0 \%$ (53/93) were female (Table 1). At THU, the single transplant was the major LTX procedure at 51.6\% (48/93). The obstructive lung disease was the most responsible for the LTX indication at $41.9 \%$ (39/93), followed by pulmonary vascular disease at $22.6 \%(21 / 93)$ and restrictive lung disease at $20.4 \%(19 / 93)$. There was no statistic difference in age, gender, LTX procedure and LTX indication between PRA+ and PRA- LTRs ( $p=$

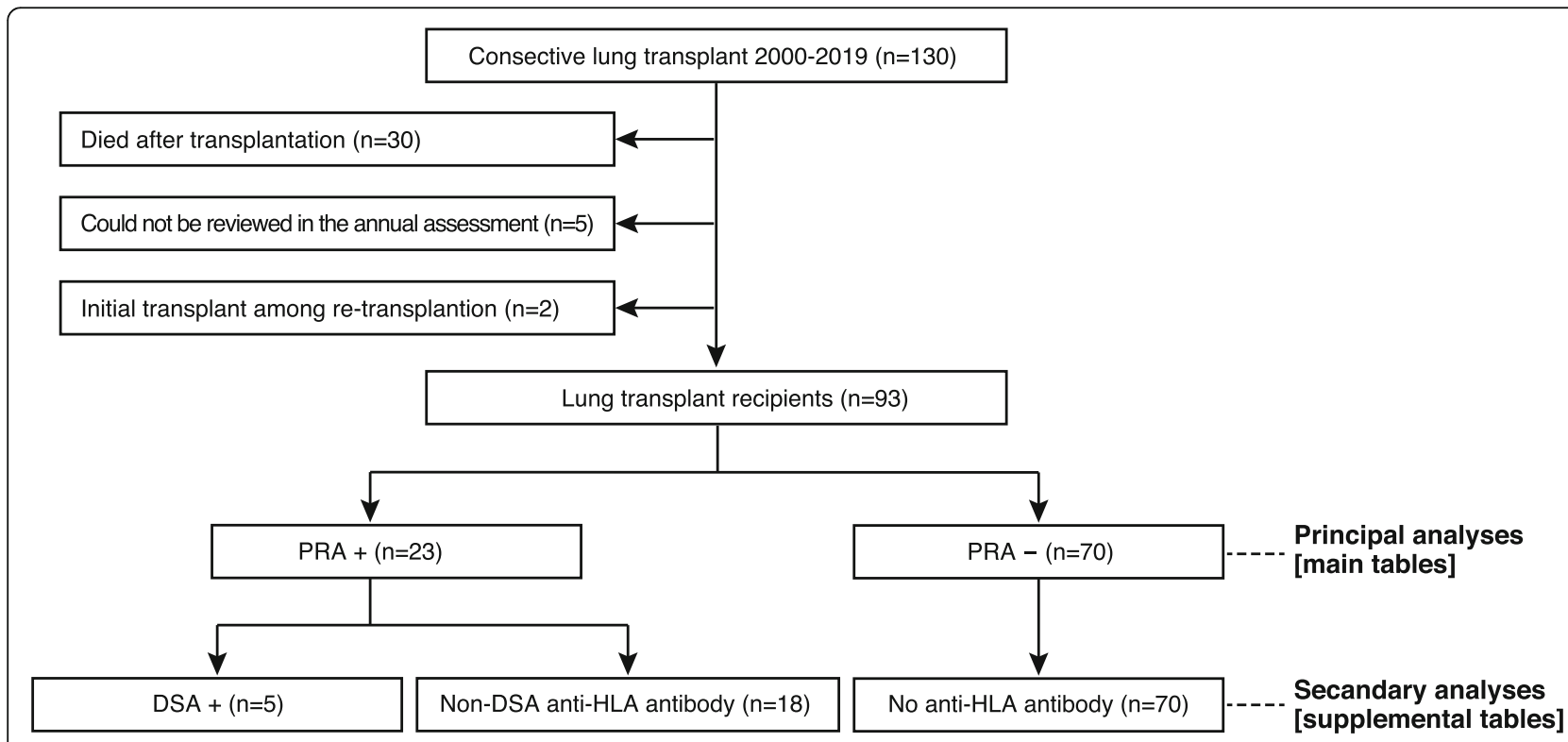

Fig. 1 Study population 
Table 1 Patients' characteristics at the time of transplant in the study $(n=93)$ and lung transplant recipients with/without PRA ( $n=23$ vs 70, respectively)

\begin{tabular}{|c|c|c|c|c|}
\hline & Total $(n=93)$ & PRA $+(n=23)$ & PRA- $(n=70)$ & $p$-value \\
\hline Age at LTX, median (IQR) & $42(32-50)$ & $45(33-50)$ & $41(31-49)$ & 0.423 \\
\hline Sex, female & $53(57.0 \%)$ & $13(56.5 \%)$ & $40(75.5 \%)$ & 1.000 \\
\hline \multicolumn{5}{|l|}{ LTX procedure } \\
\hline Single & $48(51.6 \%)$ & $11(47.8 \%)$ & 37 (52.9\%) & \multirow[t]{3}{*}{0.935} \\
\hline Double & 37 (39.8\%) & $10(43.5 \%)$ & $27(38.6 \%)$ & \\
\hline Living-donor & $8(8.6 \%)$ & $2(8.7 \%)$ & $6(8.6 \%)$ & \\
\hline \multicolumn{5}{|l|}{ LTX indication } \\
\hline Pulmonary vascular disease & $21(22.6 \%)$ & $4(17.4 \%)$ & $17(24.3 \%)$ & \multirow[t]{6}{*}{0.282} \\
\hline Restrictive lung disease & 19 (20.4\%) & $7(30.4 \%)$ & $12(17.1 \%)$ & \\
\hline Obstructive lung disease & $39(41.9 \%)$ & $7(30.4 \%)$ & $32(45.7 \%)$ & \\
\hline Suppurative lung disease & $8(8.6 \%)$ & $4(17.4 \%)$ & $4(5.7 \%)$ & \\
\hline CLAD & $2(2.2 \%)$ & $0(0 \%)$ & $2(2.9 \%)$ & \\
\hline Others & $4(4.3 \%)$ & $1(4.3 \%)$ & $3(4.3 \%)$ & \\
\hline \multicolumn{5}{|l|}{ Pre-LTX comorbidities } \\
\hline Diabetes & $6(6.5 \%)$ & $1(4.3 \%)$ & $5(7.1 \%)$ & 1.000 \\
\hline Connective tissue disease & $14(15.1 \%)$ & $6(23.1 \%)$ & $8(11.4 \%)$ & 0.102 \\
\hline GERD & $5(12.8 \%)$ & $2(8.7 \%)$ & $3(4.3 \%)$ & 0.594 \\
\hline Chronic kidney disease $\mathrm{a}^{\mathrm{a}}$ & $3(3.3 \%)$ & $1(4.3 \%)$ & $2(2.9 \%)$ & 1.000 \\
\hline
\end{tabular}

Abbreviation: PRA panel-reactive assay, IQR Interquartile range, $L T X$ lung transplant, CLAD chronic lung allograft dysfunction, GERD gastroesophageal reflux disease ${ }^{a}$ incalculable the glomerular filtration rate in 2 recipients $(n=91)$

Table 2 Transplant surgery and years since transplant in the study $(n=93)$ and lung transplant recipients with/without PRA $(n=23$ vs 70 , respectively)

\begin{tabular}{|c|c|c|c|c|}
\hline & Total $(n=93)$ & PRA $+(n=23)$ & PRA- $(n=70)$ & $p$-value \\
\hline$\overline{C M V}$ mismatch $(\mathrm{D}+/ \mathrm{R}-)^{\mathrm{a}}$ & $16(17.2 \%)$ & $1(4.3 \%)$ & $15(21.4 \%)$ & 0.058 \\
\hline Donor age\$ & & & & 0.110 \\
\hline$\leq 19$ & $9(9.7 \%)$ & $0(0 \%)$ & $9(12.9 \%)$ & \\
\hline $20-59$ & 79 (84.9\%) & $20(87.0 \%)$ & 59 (84.3\%) & \\
\hline$\geq 60$ & $4(4.3 \%)$ & $2(8.7 \%)$ & $2(2.9 \%)$ & \\
\hline Operative time $(\mathrm{min})$ & & & & 0.868 \\
\hline$\leq 419$ & $24(25.8 \%)$ & $5(21.7 \%)$ & 19 (27.1\%) & \\
\hline $420-839$ & $47(50.5 \%)$ & $12(52.2 \%)$ & $35(50.0 \%)$ & \\
\hline$\geq 840$ & $22(23.7 \%)$ & $6(26.1 \%)$ & $16(22.9 \%)$ & \\
\hline Ischemic time $(\min )^{b}$ & & & & 0.861 \\
\hline$\leq 419$ & $21(22.6 \%)$ & $5(21.7 \%)$ & $16(22.9 \%)$ & \\
\hline $420-599$ & $42(45.2 \%)$ & $9(39.1 \%)$ & $33(47.1 \%)$ & \\
\hline$\geq 600$ & $29(31.2 \%)$ & $8(34.8 \%)$ & $21(30.0 \%)$ & \\
\hline Yeas since transplant & & & & 0.048 \\
\hline$\leq 5$ years & $40(43.0 \%)$ & $14(60.9 \%)$ & $26(37.1 \%)$ & \\
\hline $5-10$ years & $36(38.7 \%)$ & $4(17.4 \%)$ & $32(45.7 \%)$ & \\
\hline$\geq 10$ years & $17(18.3 \%)$ & $5(21.7 \%)$ & $12(17.1 \%)$ & \\
\hline
\end{tabular}

Abbreviation: PRA panel-reactive assay, $C M V$ cytomegalovirus, $D$ donor, $R$ recipient

${ }^{\mathrm{a}}$ missing the CMV serology in 17 recipients $(n=76)$, \$missing the age in one donor $(n=92)$ and ${ }^{\mathrm{b}}$ missing the ischemic time in one recipient $(n=92)$ 
$0.423,1.000,0.935$ and 0.282 , respectively). Pretransplant comorbidities including diabetes (hemoglobin A1c $>6.5 \%)$, connective tissue disease, chronic kidney disease (the glomerular filtration rate $<60 \mathrm{~mL} / \mathrm{min} / 1.73$ $\mathrm{m} 2$ ) [7] and gastroesophageal reflux disease (typical symptoms and endoscopic changes) [8], were also assessed in PRA+ and PRA- groups without significance $(p=1.000,0.100,0.590$ and 1.000 , respectively). In secondary analyses, no statistic difference was found in age, gender, LTX procedure, LTX indication and pretransplant comorbidities among DSA+, non-DSA antiHLA antibody and PRA- recipients (Supplemental Table 1).

The transplant surgery was then reviewed (Table 2). Donor age $(\leq 19,20-59, \geq 60$ years old), operative time $(\leq 419,420-839, \geq 840 \mathrm{~min})$ and total ischemic time $(\leq 419,420-599, \geq 600 \mathrm{~min})$ were partitioned and compared in PRA+ and PRA- groups, without statistic difference $(p=0.09,0.900$ and 0.861 , respectively). CMV mismatch was seen less in PRA+ (4.3\%) than in PRA- recipients $(21.4 \%)$ without significance $(p=0.06)$. Recently transplanted recipients ( $\leq 5$ years post LTX) had a higher proportion of positive PRA (60.9\% (14/23)) compared to patients transplanted in earlier eras with $17.4 \%(4 / 23)$ in $5-10$ years and $21.7 \%(5 / 23)$ in $\geq 10$ years post LTX ( $p=$ $0.04)$. In secondary analyses, altogether 5 patients had DSA, $4 / 5$ (80\%) were recently transplanted while the remaining patient $(20 \%)$ was $5-10$ years post LTX. There was no positive DSA in in recipients seen $\geq 10$ years post LTX (Supplemental Table 2).

From the standpoint of HLA sensitization, pregnancy, transfusion and prior transplant are generally considered risk factors $[9,10]$. Thus, a history of pregnancy, blood transfusion and prior transplant were reviewed. This revealed no association of these factors with positive PRA $(p=0.280,0.443$ and 0.331 , respectively) (Table 3). Additionally, there was no difference in those episodes for the development of DSA (Supplemental Table 3). On the other hand, HLA-A, B and DR alleles in both recipient and donor(s) were available for analysis in 53 LTX cases, and the number of HLA mismatch was counted. The $0-2$ mismatch $(0-2 / 6$ in deceased-donor or $0-4 / 12$ in living-donor) was found in 7 LTRs, of whom 6 $(85.7 \%)$ were living-donor transplants. Meanwhile, positive DSA was seen only in the 5-6 HLA mismatch. Yet, there was no significance in the number of HLA mismatches among PRA $+/-$ recipients $(p=0.796)$ (Table 3$)$ as well as among DSA+, non-DSA anti-HLA antibody and PRA- recipients $(p=0.509)$ (Supplemental Table 3).

The lung function was assessed while the annual assessment in LTRs who survived at least 1-year post transplant $(n=84)$ (Table 4). CLAD was found in $20.2 \%$ $(17 / 84)$ of the recipients, among whom $11.8 \%(2 / 17)$ were PRA+ and $88.2 \%(15 / 17)$ were PRA- $(p=0.190)$. RAS accounted for $29.4 \%(5 / 17)$, BOS for $41.1 \%$ (7/17), mixed phenotype for $23.5 \%(4 / 17)$ and unclassified for $5.9 \%(1 / 17)$ (data not shown). In secondary analyses, no recipients diagnosed with CLAD had DSA. Antibodies in 2 sensitized recipients who were diagnosed with CLAD were non-DSA anti-HLA antibodies (Supplemental Table 4).

The detail of PRA and DSA in sensitized patients $(n=$ 23) were summarized (Table 5). Of 23 participants, the number of LTRs with sensitization only to HLA class 1 was 9 (39.1\%), of whom 3 were highly sensitized (> 25\%) $[11,12]$ and 2 have DSA (Cw4 and B52/B59). On the other hand, the number of recipients who had only antiHLA antibodies class 2 were 5 (21.7\%), of whom no one showed PRA over $25 \%$ but one recipient had DSA (DR4). Nine sensitized LTRs with anti-HLA antibodies against both HLA class 1 and 2 (39.1\%) were found, among whom 7 were highly sensitized and 2 had DSA (DR4 and DQ9). No DSA found in the study period had C1q-binding activities.

Table 3 Possible risk factors of sensitization $(n=93)$ and HLA mismatch $(n=53)$ among lung transplant recipients with/without PRA

\begin{tabular}{|c|c|c|c|c|}
\hline & Total $(n=93)$ & $\mathrm{PRA}+(n=23)$ & PRA- $(n=70)$ & $p$-value \\
\hline History of pregnant & $24(25.8 \%)$ & $8(34.8 \%)$ & $16(22.9 \%)$ & 0.280 \\
\hline History of transfusion & $84(90.3 \%)$ & $22(95.7 \%)$ & $62(88.6 \%)$ & 0.443 \\
\hline Prior transplant ${ }^{\mathrm{a}}$ & $6(6.5 \%)$ & $0(0.0 \%)$ & $6(8.6 \%)$ & 0.331 \\
\hline \multirow[t]{2}{*}{ Any episodes of acute rejection } & $13(14.0 \%)$ & $2(8.7 \%)$ & $11(15.7 \%)$ & 0.508 \\
\hline & Total $(n=53)$ & $\mathrm{PRA}+(n=13)$ & PRA- $(n=40)$ & $p$-value \\
\hline HLA mismatch $A / B / D R^{b}$ & & & & 0.796 \\
\hline mismatch 0-2 & $7(13.2 \%)$ & $1(7.7 \%)$ & $6(15.0 \%)$ & \\
\hline mismatch 3-4 & $23(43.4 \%)$ & $6(46.2 \%)$ & $17(42.5 \%)$ & \\
\hline mismatch 5-6 & $23(43.4 \%)$ & $6(46.2 \%)$ & $17(42.5 \%)$ & \\
\hline
\end{tabular}

Abbreviation: PRA panel-reactive assay, HLA human leukocyte antigen

a including solid organ transplant and hematopoietic stem cell transplantation prior to lung transplantation

${ }^{b}$ the number of mismatched HLA-A/B/DR alleles (0-6) between donors and recipient in deceased-donor transplant and the total number of mismatch alleles (012) were divided to half in living-donor transplant 
Table 4 The association of CLAD with sensitization in those who survived one year after lung transplant $(n=84)$

\begin{tabular}{lllll}
\hline & Total $(\boldsymbol{n}=\mathbf{8 4})$ & CLAD- $(\boldsymbol{n}=\mathbf{6 7})$ & $\mathrm{CLAD}+(\boldsymbol{n}=\mathbf{1 7})$ & $\boldsymbol{p}$-value \\
\hline PRA- & $64(76.2 \%)$ & $49(73.1 \%)$ & $15(88.2 \%)$ & 0.191 \\
PRA+ & $20(23.8 \%)$ & $18(26.9 \%)$ & $2(11.8 \%)$ & \\
\hline
\end{tabular}

Abbreviation: PRA panel-reactive assay, CLAD chronic lung allograft dysfunction

\section{Discussion}

The status of HLA-sensitization post transplantation was reviewed in a lung transplant center in Japan. Somewhat surprisingly, only $24.7 \%$ (23/93) Japanese recipients were PRA positive. Even fewer recipients had DSA $(5.4 \%, 5 / 93)$, which was far lower than that of other transplant facilities, e.g. over $80 \%$ of 320 lung transplant recipients were sensitized and around a half had developed DSA within 2 years post-transplantation in Toronto, Canada [2], 50.1\% (105/206) of recipients transplanted within a year had DSA in Suresnes, France [3] and 63.9\% (124/194) in LTRs developed DSA in Dallas, United States [13]. However, the data shown in this study is a snapshot of a single and often remote time point from the LTX operation. Therefore, careful attention should be paid in interpreting our result with low prevalence of sensitization and DSA in our cohort.

Throughout the study, more sensitized LTRs were found in recent transplantations ( $\leq 5$ years post LTX) than the earlier ( $5-10$ years or $\geq 10$ years post LTX), and the majority of recipients who carried DSA were the recent cases but not in the older ones. This can be possible survival bias that some of earlier recipients may have died owing to graft failure without ever knowing about DSA and we thus have seen less sensitized recipients in the older cohort. To further elucidate the possible bias, the cause of death was reviewed among those who died after transplant $(n=30)$ (Supplemental Table 5). Of those, 24 were found with an obvious cause of death which was highly unlikely to be related to antibodymediated rejection (AMR). As transplantation was not scheduled when there was a positive CDC crossmatch, humoral rejection due to pre-existing DSA (hyper-acute rejection) was also unlikely in cases with primary graft dysfunction. Presence of DSA could not be denied in 6 recipients, of whom 3 CLAD cases and 1 unknown were transplanted in the earlier ( $>5$ years). Even in the scenario where those 4 LTRs may have died with AMR, the influence of DSA to death is still small in those population. Given the low incidence of sensitization, long survival and little impact of DSA to death, it is conceivable that the earlier recipients were less sensitized, with which the survival bias may be implausible. Instead, this raised the assumption that positive PRA or DSA could be higher in the recent cases but not in the older cohort in the population.

This could be explained if recipients surviving $>5$ years post LTX might have transiently developed DSA that disappeared thereafter and are currently recognized as unsensitized. The study done in Houston, United States [14] indicated that transient de novo DSA was commonly seen and associated with a lower risk of postLTX acute rejection than that of persistent de novo DSA. Similarly, the study from Munich, Germany [15]

Table 5 The detail of PRA and DSA in the study $(n=93)$

\begin{tabular}{|c|c|c|c|c|}
\hline & & \multicolumn{3}{|l|}{ DSA } \\
\hline & & Identification & MFI & C1q-binding \\
\hline PRA class $1+/$ class2- & 9 & & & \\
\hline PRA $>25 \%$ & 3 & & & \\
\hline Non-DSA anti-HLA antibodies & 7 & & & \\
\hline \multirow[t]{2}{*}{ DSA (MFI > 1000) } & 2 & Cw4 & 1243 & negative \\
\hline & & B52, B59 & 4249,2860 & negative \\
\hline PRA class 1-/class2+ & 5 & & & \\
\hline PRA $>25 \%$ & 0 & & & \\
\hline Non-DSA anti-HLA antibodies & 4 & & & \\
\hline DSA (MFI > 1000) & 1 & DR4 & 1248 & negative \\
\hline PRA class $1+/$ class $2+$ & 9 & & & \\
\hline PRA $>25 \%$ & 7 & & & \\
\hline Non-DSA anti-HLA antibodies & 7 & & & \\
\hline \multirow[t]{2}{*}{ DSA (MFI > 1000) } & 2 & DR4 & 1137 & negative \\
\hline & & DQ9 & 4091 & negative \\
\hline
\end{tabular}

Highly sensitized PRA was defined over $25 \%$

Abbreviation: PRA panel-reactive antibody, DSA donor-specific antibody, MFI mean fluorescence intensity 
showed that persistent DSA was associated with reduced survival compared with transient DSA. Given these facts, our earlier cases might have had transient DSA and currently undetectable DSA, and thus show longer survival. However, it is unclear from the study design whether LTRs in our center have had DSA before or after transplant since PRA and DSA are time-dependent variables. To make those above questions more clear, a longitudinal study with repeated measurement of PRA and DSA before and after transplantation is needed. Yet, this study should be fundamental for future trials in Japan as it underlines our need to better understand our LTX population.

Based on the National Marrow Donor Program [16], HLA allele frequency varies by race and ethnicity in the US Population. With those features in mind, it should be interesting to evaluate the status of sensitization and DSA frequency in the Japanese population with a largescale analysis as they have relatively little genetic diversity, with the notable exception of the Okinawa region [17]. In our center, the 0-2 HLA mismatch was only seen in living-donor LTX recipients who were PRA negative (Table 3), while positive DSA was seen only in the 5-6 HLA mismatch. However, it is inconclusive whether the number of mismatches is associated with HLA-sensitization as the sample size was too small to show statistical difference in the study. Meanwhile, it is worthwhile to see the HLA-DQ mismatches in the population that drives de novo DSA in LTX [18]. Despite our data, de novo DSA are common after LTX and associated with an increased risk of CLAD. However, HLA typing at DQ loci is not mandatory for transplant matching for the time being and has not been routinely investigated in Japan. To pursue the role of DQ matching between a recipient and donors, consecutive measurement of HLA loci will be required in the next study.

Given the cross-sectional analysis in a single-center, we have several limitations that warrant discussion. First, the number of LTX recipients is still in short for analysis. Despite the transplant circumstance changing in Japan, the shortage of organ donors is one of the largest issues in all solid organ transplantation [1-19]. Analysis was done in sensitized vs non-sensitized recipients, rather than positive vs negative DSA, due to the small sample number. To overcome this, the multicenter study would benefit from seeing the impact of DSA in Japanese population. Second, a universal health care insurance system in Japan began to cover the fee of anti-HLA antibody among LTX recipients in April 2018. Thus, little has been known about the status of sensitization in LTX candidates when registered and that of DSA in LTRs throughout the country. The successive measurement of DSA in LTR could be a fundamental data to analyze the relationship of de novo DSA to CLAD.
Although some limitations are need to be addressed, this is the first report documenting the sensitization status and DSA in LTX recipients, so that provides valuable tip to other transplant centers in Japan.

\section{Conclusions}

To sum up, the rate of PRA positive LTRs in the Japanese population was lower compared with those in previous reports from US and Europe. More sensitized LTRs were found in recent transplantations than the older cohort, and DSA was identified in the recent recipients. Due to several limitations, it is still unclear whether the sensitization would be related to the development of CLAD or survival, yet this study would be fundamental to the future anti-HLA body study in Japanese population.

\section{Supplementary information}

Supplementary information accompanies this paper at https://doi.org/10. 1186/s12890-020-01299-0.

Additional file 1: Supplemental Table 1. Patients' characteristics at the time of transplant and lung transplant recipients with/without DSA ( $n=93$ ). Supplemental Table 2. Transplant surgery and years since transplant in lung transplant recipients with/without DSA $(n=93)$. Supplemental Table 3. Possible risk factors of sensitization $(n=93)$ and HLA mismatch $(n=53)$. Supplemental Table 4. The association of CLAD with/without DSA in those who survived one year after lung transplant $(n=84)$. Supplemental Table 5. Cause of death and possible antibody mediated rejection among transplant patients who have passed away $(n=30)$.

\section{Abbreviations}

BE: Bronchiectasis; BOS: Bronchiolitis obliterans syndrome; CLAD: Chronic lung allograft rejection; COPD: Chronic obstructive pulmonary disease; CKD: Chronic kidney disease; IPF: Idiopathic pulmonary fibrosis; IP: Interstitial pneumonia; LTX: Lung transplant; LTR: Lung transplant recipient;

$\mathrm{PAH}$ : Pulmonary arterial hypertension; RAS: Restrictive allograft syndrome

\section{Acknowledgements}

Not applicable.

\section{Authors' contributions}

SK and TH are the guarantors of this manuscript. TH has full access to all of the data in the study and gathered information from the database. $\mathrm{YW}, \mathrm{HO}$, $\mathrm{HN}, \mathrm{MA}$ and JT contributed substantially to the study design, the data analysis and interpretation, and wrote the manuscript. TH is responsible for statistical analysis. YO had full access to all of the data in the study and takes responsibility for the integrity of the data and the accuracy of the data analysis. All authors have read and approved the manuscript.

\section{Funding}

The collection, analysis, and interpretation of data were supported in a part by Takeda Science Foundation, Kurozumi Medical Foundation and Grant-inAid for Scientific Research C (20 K08509). No additional external funding was received for this study.

\section{Availability of data and materials}

The datasets used and/or analysed during the current study are available from the corresponding author on reasonable request.

\section{Ethics approval and consent to participate}

The study protocol was reviewed by the Institutional Review Board (IRB) at Tohoku University Hospital (IRB number 2019-01-54). In light of the 
retrospective design, the requirement of informed consent was waived. We disclosed information on the implementation of the research and ensured the opportunity for research subjects to refuse participation by posting the information disclosure materials approved by the Ethics Committee on the website of the Graduate School of Medicine, Tohoku University.

\section{Consent for publication}

Not applicable.

\section{Competing interests}

The authors declare that they have no competing interests.

\section{Author details}

'Department of Thoracic Surgery, Institute of Development, Aging and Cancer, Tohoku University, Sendai, Miyagi, Japan. ${ }^{2}$ Division of Organ Transplantation, Tohoku University Hospital, 4-1 Seiryomachi, Sendai, Miyagi 980-8574, Japan. ${ }^{3}$ Multi-Organ Transplant Program, Toronto General Hospital, Toronto, ON, Canada.

Received: 17 April 2020 Accepted: 21 September 2020

Published online: 02 October 2020

\section{References}

1. Date H. Current status and problems of lung transplantation in Japan. J Thorac Dis. 2016:8(Suppl 8):S631-6.

2. Tikkanen JM, Singer LG, Kim SJ, Li Y, Binnie M, Chaparro C, et al. De novo DQ donor-specific antibodies are associated with chronic lung allograft dysfunction after lung transplantation. Am J Respir Crit Care Med. 2016; 194(5):596-606.

3. Roux A, Le Lan IB, Holifanjaniaina S, Thomas KA, Picard C, Grenet D, et al. Characteristics of donor-specific antibodies associated with antibodymediated rejection in lung transplantation. Front Med. 2017:4(OCT):1-10.

4. Verleden SE, Vanaudenaerde BM, Emonds M-P, Van Raemdonck DE, Neyrinck AP, Verleden GM, et al. Donor-specific and -nonspecific HLA antibodies and outcome post lung transplantation. Eur Respir J. 2017;50(5): 1701248.

5. Verleden GM, Glanville AR, Lease ED, Fisher AJ, Calabrese F, Corris PA, et al. Chronic lung allograft dysfunction: definition, diagnostic criteria, and approaches to treatment-a consensus report from the pulmonary council of the ISHLT. J Heart Lung Transplant. 2019;38(5):493-503.

6. Glanville AR, Verleden GM, Todd JL, Benden C, Calabrese F, Gottlieb J, et al. Chronic lung allograft dysfunction: Definition and update of restrictive allograft syndrome-A consensus report from the Pulmonary Council of the ISHLT. J Heart Lung Transplant. 2019;38(5):483-92 Available from.

7. National Institute for Health and Care Excellence. Chronic kidney disease in adults: assessment and management [CG182]. London; 2014. https://www. nice.org.uk/quidance/cg182.

8. Katz PO, Gerson LB, Vela MF. Guidelines for the diagnosis and management of gastroesophageal reflux disease. Am J Gastroenterol. 2013;108(3):308-28 quiz 329.

9. Hyun J, Park KD, Yoo Y, Lee B, Han BY, Song EY, et al. Effects of different sensitization events on HLA alloimmunization in solid organ transplantation patients. Transplant Proc. 2012;44(1):222-5.

10. Abbes S, Metjian A, Gray A, Martinu T, Snyder L, Chen D, et al. Human leukocyte antigen sensitization in solid organ transplantation: a primer on terminology, testing, and clinical significance for the apheresis practitioner. Ther Apher Dial. 2017;21(5):441-50.

11. Aversa M, Benvenuto L, Kim H, Shah L, Robbins H, Payne Stanifer B, et al. Effect of calculated panel reactive antibody value on waitlist outcomes for lung transplant candidates. Ann Transplant. 2019;24:383-92.

12. Hadjiliadis D, Chaparro C, Reinsmoen NL, Gutierrez C, Singer LG, Steele MP, et al. Pre-transplant panel reactive antibody in lung transplant recipients is associated with significantly worse post-transplant survival in a multicenter study. J Hear Lung Transplant. 2005;24(7 SUPPL):249-54.

13. Sullivan D, Ahn C, Gao A, Lacelle C, Torres F, Bollineni S, et al. Evaluation of current strategies for surveillance and management of donor-specific antibodies: single-center study. Clin Transpl. 2018:32(7):1-11.

14. Islam AK, Sinha N, DeVos JM, Kaleekal TS, Jyothula SS, Teeter LD, et al. Early clearance vs persistence of de novo donor-specific antibodies following lung transplantation. Clin Transpl. 2017;31(8):1-9.
15. Schmitzer $M$, Winter $H$, Kneidinger $N$, Meimarakis $G$, Dick A, Schramm $R$, et al. Persistence of de novo donor-specific HLA-antibodies after lung transplantation: a potential marker of decreased patient survival. Hla. 2018; 92(1):24-32.

16. Maiers M, Gragert L, Klitz W. High-resolution HLA alleles and haplotypes in the United States population. Hum Immunol. 2007:68(9):779-88.

17. Yamaguchi-Kabata Y, Nakazono K, Takahashi A, Saito S, Hosono N, Kubo M, et al. Japanese population structure, based on SNP genotypes from 7003 individuals compared to other ethnic groups: effects on population-based association studies. Am J Hum Genet. 2008;83(4):445-56.

18. McCaughan JA, Battle RK, Singh SKS, Tikkanen JM, Moayedi Y, Ross HJ, et al. Identification of risk epitope mismatches associated with de novo donorspecific HLA antibody development in cardiothoracic transplantation. Am J Transplant. 2018;18(12):2924-33.

19. Akabayashi A, Nakazawa E, Ozeki-Hayashi R, Tomiyama K, Mori K, Demme RA, et al. Twenty years after enactment of the organ transplant law in Japan: why are there still so few deceased donors? Transplant Proc. 2018; 50(5):1209-19.

\section{Publisher's Note}

Springer Nature remains neutral with regard to jurisdictional claims in published maps and institutional affiliations.
Ready to submit your research? Choose BMC and benefit from:

- fast, convenient online submission

- thorough peer review by experienced researchers in your field

- rapid publication on acceptance

- support for research data, including large and complex data types

- gold Open Access which fosters wider collaboration and increased citations

- maximum visibility for your research: over $100 \mathrm{M}$ website views per year

At $\mathrm{BMC}$, research is always in progress.

Learn more biomedcentral.com/submissions 